\section{Cahiers de Narratologie}

Analyse et théorie narratives

$14 \mid 2008$

Prose d'idées : formes et savoirs

\title{
Raphaël Baroni, La tension narrative. Suspense, curiosité, surprise, Paris, Seuil, 2007
}

\section{Lorenzo Bonoli}

\section{(2) OpenEdition}

1 Journals

Édition électronique

URL : http://journals.openedition.org/narratologie/608

DOI : 10.4000/narratologie.608

ISSN : 1765-307X

Éditeur

LIRCES

\section{Référence électronique}

Lorenzo Bonoli, «Raphaël Baroni, La tension narrative. Suspense, curiosité, surprise, Paris, Seuil, 2007 », Cahiers de Narratologie [En ligne], 14 | 2008, mis en ligne le 06 mars 2008, consulté le 21 septembre 2020. URL : http://journals.openedition.org/narratologie/608 ; DOI : https://doi.org/10.4000/ narratologie.608

Ce document a été généré automatiquement le 21 septembre 2020.

\section{c) (†) $९$}

Cahiers de Narratologie - Analyse et théorie narratives est mis à disposition selon les termes de la licence Creative Commons Attribution - Pas d'Utilisation Commerciale - Pas de Modification 4.0 International. 


\title{
Raphaël Baroni, La tension narrative. Suspense, curiosité, surprise, Paris, Seuil, 2007
}

\author{
Lorenzo Bonoli
}

1 Comment se fait-il que nous aimions rester "suspendus aux lèvres d'un conteur ", que nous nous retrouvions «incapables d'interrompre la lecture d'un livre qui nous passionne » ou encore que nous soyons «tenus en haleine par un film» (p. 17) ? Et, surtout, de quelle façon les récits suscitent-ils et entretiennent-ils une telle tension chez l'auditeur, le lecteur ou le spectateur? Voici les questions centrales qui accompagnent la réflexion de Raphaël Baroni dans son livre La tension narrative et qui le conduisent à proposer une nouvelle approche du récit et de son fonctionnement, non plus limitée à une analyse des structures textuelles qui le composent, mais centrée sur l'effet esthétique et affectif qu'il suscite chez son interprète.

2 Une telle thématique, bien qu'intuitivement centrale pour comprendre le rôle joué par les récits dans nos vies, a été longtemps négligée par les recherches en narratologie et en critique littéraire, surtout pendant la période structuraliste où la célèbre «fermeture du texte» n'encourageait pas les chercheurs à s'aventurer au-delà de l'étude des structures immanentes des récits, de leur composition et des rapports entre action réelle et action représentée.

3 La réflexion de Baroni, tout en s'inscrivant dans le prolongement de l'important héritage structuraliste, ose enfin se focaliser sur le thème des effets affectifs suscités par les récits en complétant la question traditionnelle : « quelles structures composent les récits?» avec une interrogation sur leur fonctionnement et leurs effets sur l'interprète: "comment se fait-il que nous soyons 'pris' ou 'affectés' par ces structures? ». La réflexion théorique se confronte, dans les derniers chapitres, à des exemples tirés de systèmes sémiotiques variés : la publicité, la bande dessinée (Tardi), le cinéma (avec l'analyse d'une étude de Sylvain Rigollot de la tension narrative dans Titanic), un conte de Grimm, et quelques nouvelles de Borges. 
4 L'enjeu du livre de Baroni est de comprendre et de décrire la « tension » entretenue par l'indétermination provisoire qui caractérise les récits à intrigue en la faisant émerger en tant qu'effet esthétique - de la réponse interprétative du lecteur ou du spectateur confronté aux structures narratives et en soulignant que cette réponse interprétative ne se situe pas uniquement à un niveau cognitif, mais convoque également une dimension affective qui suscite des passions et des émotions.

5 Il est cependant évident qu'une telle problématique, avec la nouvelle approche du récit qu'elle implique, exige un élargissement du cadre disciplinaire, les outils théoriques traditionnels de l'analyse des récits étant mal adaptés pour parler de notions telle que le plaisir, le suspense, la surprise, etc. D'où la nécessité de convoquer dans la réflexion, à côté des références disciplinaires habituelles - linguistique, sémiotique, rhétorique, etc. - d'autres références telles que la pragmatique, la psychologie cognitive, la psychologie affective, les théories de l'action et l'interactionnisme, etc.

6 C'est à ce niveau que le livre de Baroni réussit pleinement son pari d'aborder le thème délicat de la tension narrative en constituant un cadre théorique cohérent où s'intègrent les instruments traditionnels de l'analyse des récits et des éléments nouveaux venant des disciplines qui viennent d'être évoquées. A l'intérieur de ce nouveau cadre, il devient enfin possible de parler avec rigueur et précision, non seulement de la structuration des récits et de leur mise en intrigue, mais aussi de notions telles que le plaisir, le suspense, la curiosité ou la surprise, qui créent souvent un certain embarras parmi les chercheurs.

7 Certes, la constitution d'un tel cadre théorique n'a pas été facile en raison surtout d'un saut épistémologique qui sépare les analyses structuralistes - portant sur les textes dans leur autonomie, indépendamment du sujet qui le lit - et les notions de plaisir ou d'affects en général - qui, quant à elles, renvoient à un individu qui fait une expérience de lecture particulière. Baroni réussit à résoudre ce problème en faisant référence aux théories de la lecture qui se sont développées à partir de la fin des années 70 . Ces théories offrent une première tentative de dépasser la fermeture du texte structuraliste à travers la dichotomie entre lecteur modèle ou implicite et lecteur empirique ou réel, elles proposent ainsi d'articuler une dimension structurale avec une dimension subjective et individuelle. Baroni complète en outre l'apport de ces théories en intégrant à son approche une série de recherches en psychologie cognitive et affective qui précisent le fonctionnement de la réception des récits du point de vue du sujet lisant et offrent en même temps des outils indispensables pour penser la dimension affective suscitée par la lecture.

8 Ainsi, le mérite principal du livre de Baroni est sûrement celui d'avoir osé dépasser les frontières disciplinaires traditionnelles de l'analyse des récits pour constituer un nouveau cadre de référence cohérent qui permette enfin de parler du plaisir de la lecture, de son suspense ou de ses surprises; et il n'est pas exclu que ce cadre de référence puisse devenir, comme le laisse entendre Jean-Marie Schaeffer dans la préface de l'ouvrage, un nouveau paradigme dans les études narratologiques à venir.

9 Reste encore un élément à souligner qui fait surface à plusieurs reprises dans le livre et qui pourrait sembler somme toute marginal dans la réflexion narratologique proposée, mais qui en fait revêt une certaine importance pour comprendre la portée des implications de celle-ci. Baroni en effet inscrit sa réflexion narratologique du fonctionnement du récit dans une réflexion d'ordre philosophique sur la fonction anthropologique du récit et plus précisément de la tension narrative. D’une part, en 
s'inscrivant dans le prolongement des thèses de Paul Ricœur dans Temps et Récit, Baroni souligne le rôle que la tension narrative joue dans la représentation de la temporalité propre à l'existence humaine. $\mathrm{Si}$, comme le soutient Ricœur, le récit a bien quelque chose à voir avec la manière dont nous faisons l'expérience de notre temporalité :

Cette profondeur temporelle n'apparaît jamais avec autant d'éclat que dans l'incertitude anticipatrice qu'éprouve l'interprète durant l'expérience esthétique, dans ce suspense ou cette curiosité qui font la force des intrigues fictionnelles (p.

18)

10 Par ailleurs, cette "incertitude anticipatrice » nous révèle une deuxième dimension anthropologique de la tension narrative qui nous renvoie à l'expérience des limites du savoir qui peut être construit par l'homme et, finalement, à sa finitude existentielle. Le récit permet en effet :

de mettre en scène, d'une part, l'incertitude du futur tissé de projet, d'espoirs ou de craintes et, d'autre part, l'aspect sous-déterminé de notre rapport au monde ; dans cette dernière variante, l'intrigue souligne (malgré nos diagnostics incessants) notre incapacité à lire les pensées d'autrui, à saisir les intentions cachées derrières les gestes, à comprendre les événements dans lesquels nous sommes enchevêtrés, à ressaisir un passé irrémédiablement opaque. (p. 406)

11 Ces dernières considérations me permettent enfin de souligner l'ampleur de la réflexion proposée par Baroni dans ce livre et les nombreuses pistes de lecture qui le traversent. Celles-ci peuvent en effet suivre aussi bien le fil des analyses de la composition de la séquence narrative ou de la présentation du schématisme de l'intrigue, que celui du fonctionnement de la participation interprétative ou des aspects plus psychologiques de la dimension affective de cette participation, en s'aventurant jusqu'à une réflexion philosophique sur la fonction anthropologique de la tension narrative. Une telle ampleur n'est pas seulement le symptôme d'une réflexion pionnière qui a dû explorer de nombreuses pistes avant de déterminer son chemin, mais aussi le reflet de l'intention de l'auteur d'ouvrir l'étude du récit à l'ensemble de ses implications linguistique, narratologique, psychologique et philosophique. 